\title{
Anti-inflammatory effect of elemental diets with different fat composition in experimental colitis
}

\author{
E. Papada ${ }^{1}$, A. C. Kaliora ${ }^{2}$, A. Gioxari ${ }^{2}$, A. Papalois ${ }^{3}$ and A. Forbes ${ }^{1 *}$ \\ ${ }^{1}$ Centre for Gastroenterology and Clinical Nutrition, University College London, Rockefeller Building, Gower Street, London \\ WC1E 6BT, UK \\ ${ }^{2}$ Department of Science of Dietetics-Nutrition, Harokopio University, 70 El. Venizelou Str., 17671 Kallithea, Athens, Greece \\ ${ }^{3}$ Experimental Research Unit, ELPEN-Pharmaceuticals Company, Inc., Pikermi Attikis, Greece \\ (Submitted 16 April 2013 - Final revision received 8 July 2013 - Accepted 8 October 2013 - First published online 14 November 2013)
}

Abstract

The aim of the present study was to evaluate the effectiveness of two isoenergetic elemental formulae with different fat content in the rat model of trinitrobenzene sulphonic acid (TNBS) colitis that mimics human inflammatory bowel disease. A total of forty-five male Wistar rats were assigned to five groups: (1) control group; (2) TNBS-induced colitis group; (3) TNBS-induced colitis group fed a long-chain TAG (LCT)-rich diet; (4) TNBS-induced colitis group fed a medium-chain TAG (MCT)-rich diet; (5) TNBS-induced colitis group fed a baseline diet and administered infliximab. Nutritional management lasted $12 \mathrm{~d}$ before and $4 \mathrm{~d}$ after rectal administration of TNBS. Subsequently, the rats were killed, and colonic tissue samples were collected for the assessment of histology, inflammation and oxidative stress. The MCT-rich diet decreased IL-6, IL-8 and intercellular adhesion molecule-1 (ICAM-1) levels and glutathione $S$-transferase (GST) activity, while the LCT-rich diet reduced only ICAM-1 levels and GST activity $(P<0 \cdot 05)$. Neither elemental formula affected IL-10 levels. Infliximab reduced IL-8 and ICAM-1 levels and GST activity and increased IL-10 levels $(P<0.05)$. No significant differences were detected in oxidative stress. Histological damage scores differed significantly only between the control and the TNBS-induced colitis group. A MCT-rich formula seems to exert stronger anti-inflammatory effects than a LCT-rich formula in TNBS colitis.

\section{Key words: Inflammatory bowel disease: Elemental formulae: Medium-chain TAG: Immunomodulation}

Inflammatory bowel disease (IBD), including Crohn's disease (CD) and ulcerative colitis, is characterised by chronic inflammation of the gastrointestinal tract and by periods of relapse and remission. IBD patients are also at a higher risk of colorectal cancer. The incidence rates of IBD are between three and fifteen cases per 100000 persons in the Western world, but there is also an increasing trend in low-incidence regions ${ }^{(1,2)}$.

A combination of genetic, environmental and immunological factors contributes to the pathogenesis of IBD. It seems that an abnormal immune response to the normal microflora of the gut and an imbalance between pro-inflammatory and anti-inflammatory factors underlie the clinical features. The excessive inflammatory response includes the expression of pro-inflammatory cytokines such as TNF- $\alpha$, IL-1, IL-6, and IL-8 and adhesion molecules such as intercellular adhesion molecule-1 (ICAM-1) and vascular cell adhesion molecule-1 $(\mathrm{VCAM}-1)^{(3,4)}$. Oxidative stress is also present due to the excessive presence of oxidised molecules and the gradual depletion of antioxidant mechanisms. As a consequence, lipid peroxidation and oxidative DNA damage are increased in IBD patients ${ }^{(5,6)}$.

Medical management aims to induce and maintain remission with the use of drugs such as aminosalicylates (e.g. sulfasalazine), corticosteroids (e.g. hydrocortisone), immunomodulators (e.g. azathioprine), antibiotics (e.g. metronidazole) and biological agents acting against TNF- $\alpha$ (e.g. infliximab) ${ }^{(7)}$. These biological agents are effective both in the induction of remission and in the preservation/recovery of the intestine ${ }^{(8)}$.

However, since the currently used therapies are not always successful and may cause important side effects, there is an increasing interest in the role of nutritional therapy in the management of IBD. In particular, defined enteral nutrition (EN) seems to be able to play a critical role in the induction and maintenance of remission through mucosal healing and decrease of pro-inflammatory cytokine levels ${ }^{(9)}$. The optimal

Abbreviations: BW, body weight; CD, Crohn's disease; EN, enteral nutrition; GST, glutathione S-transferase; IBD, inflammatory bowel disease; ICAM-1, intercellular adhesion molecule-1; LCT, long-chain TAG; MCT, medium-chain TAG; TNBS, trinitrobenzene sulphonic acid. 
composition of a therapeutic EN formula and, especially, the role of fat type in the effectiveness of EN remain controversial. Regarding the amount of long-chain TAG (LCT), some studies have shown a negative effect from increased LCT content $^{(10,11)}$, while a meta-analysis has shown no difference between high- and low-LCT formulae ${ }^{(12)}$. However, LCT induce a greater inflammatory response than medium-chain TAG (MCT) ${ }^{(13)}$, thus suggesting that MCT may be preferable, and in a rat colitis model, MCT supplementation has been shown to result in decreased inflammation in the intestinal mucosa ${ }^{(14,15)}$. In addition, $n-6$ and $n-3$ PUFA have different impacts on inflammatory mechanisms, and some experimental studies of colitis have shown the beneficial effects of $n$ - 3 PUFA on inflammation, colonic damage and oxidative stress ${ }^{(16,17)}$. Prompted by these data and the debated role of fat type in the nutritional management of IBD, the aim of the present study was to assess the effects of two commercially available isoenergetic EN formulae with different fat composition (MCT and LCT; $n-6: n-3$ ratio) on a rat model of colitis that resembles human IBD.

\section{Experimental methods}

\section{Materials}

Trinitrobenzene sulphonic acid (TNBS), butylated hydroxytoluene, PBS and Bradford reagent were obtained from Sigma-Aldrich Company. Xylazine was obtained from Pfizer Company. Ketamine and pentobarbital (Dolethal ${ }^{\circledR}$ ) were purchased from Vétoquinol Company. All the ELISA kits used for the determination of ICAM-1, IL-6, IL-8 and IL-10 levels were obtained from R\&D Systems. The malondialdehyde reagent kit was obtained from OxisResearch. The glutathione $S$-transferase (GST) assay kit was purchased from IBL International GmbH. Emsogen ${ }^{\circledR}$ and Elemental 028 Extra ${ }^{\circledR}$ were purchased from Nutricia Limited.

\section{Experimental animals and diets}

Adult male Wistar rats were obtained from the Greek Pasteur Institute and were kept in stainless steel cages at constant environmental conditions $\left(22^{\circ} \mathrm{C}\right.$ temperature and $50 \%$ humidity) under a $12 \mathrm{~h}$ day $-12 \mathrm{~h}$ night cycle. An acclimatisation period of 1 week preceded the experiment, during which all the rats were fed a standard pelleted diet (Vergerio Mangimi srl), the composition of which is given in Table 1. Access to food and water was free. The study was approved by the Animal Care Committee and was in agreement with the European Union Act and Greek Law 160, A-64, May 1991.

Table 1. Composition of the oral pelleted diet

\begin{tabular}{lc}
\hline & Pellets \\
\hline Energy $(\mathrm{kJ} / 100 \mathrm{~g})$ & 1729 \\
Protein $(\mathrm{g})$ & 19 \\
Carbohydrate $(\mathrm{g})$ & 78.5 \\
Fat $(\mathrm{g})$ & 2.5 \\
\hline
\end{tabular}

Table 2. Composition of the elemental diets

\begin{tabular}{lcc}
\hline & $\begin{array}{c}\text { LCT-rich diet } \\
\left.\text { (Elemental 028 Extra }{ }^{\circledR}\right)\end{array}$ & $\begin{array}{c}\text { MCT-rich diet } \\
\left(\text { Emsogen }^{\circledR}\right)\end{array}$ \\
\hline Energy (kJ/100 g powder) & 1854 & 1833 \\
Protein equivalent (g) & $12 \cdot 5$ & 12.5 \\
Total amino acids (g) & 15 & 15 \\
Carbohydrate (g) & 59 & 60 \\
Fat (g) & $17 \cdot 45$ & 16.4 \\
SFA (g) & $6 \cdot 7$ & 13.2 \\
MUFA (g) & $7 \cdot 4$ & 0.6 \\
PUFA (g) & $2 \cdot 4$ & 1.7 \\
\%MCT & 35 & 83 \\
\%LCT & 65 & 17 \\
$n-6: n$-3 & $4: 1$ & $46.5: 1$ \\
Energy from LA & 4 & 3.4 \\
Energy from ALA & 1 & 0.07 \\
\hline
\end{tabular}

LCT, long-chain TAG; MCT, medium-chain TAG; LA, linoleic acid; ALA, $\alpha$-linolenic acid.

A total of forty-five rats weighing 413.9 (SEM 17.2) g were randomly assigned to five groups of nine rats as follows: group A - control, healthy, untreated rats; group B - TNBSinduced colitic rats; group C - TNBS-induced colitic rats fed Elemental 028 Extra $^{\circledR}$; group D - TNBS-induced colitic rats fed Emsogen ${ }^{\circledR}$; group E - TNBS-induced colitic rats treated with infliximab.

Rats in groups A, B and E continued to be fed the baseline pelleted diet. Rats in groups $\mathrm{C}$ and $\mathrm{D}$ were fed elemental diets diluted in water $(2: 1)$ for $12 \mathrm{~d}$ before the induction of colitis and during the measurement of TNBS activity thereafter. The composition of the elemental diets is given in Table 2. Overall, the energy, protein, carbohydrate and fat content was virtually the same. In terms of fat composition, one elemental diet contained more LCT (65\%) and less MCT (35\%) and had a ratio of $n$-6:n-3 PUFA of $4: 1$, while another elemental diet was higher in MCT (83\%) than in LCT (17\%) and had a higher ratio of $n-6: n-3$ PUFA $(46 \cdot 5: 1)$.

\section{Induction of trinitrobenzene sulphonic acid colitis}

On day 0 , the rats were weighed. All the rats, except those in group A, were placed in a cage with $8 \%$ sevoflurane for $1 \mathrm{~min}$ to cause mild anaesthesia and muscle relaxation. Next, TNBS solution $(100 \mathrm{mg} / \mathrm{kg}$ body weight (BW)) in $0.25 \mathrm{ml}$ of $50 \%$ ethanol was administered intracolonically through a venoustype catheter. The rats were kept in a vertical, head-down position for $2 \mathrm{~min}$ to prevent leakage. Rats in group $\mathrm{E}$ were injected subcutaneously with infliximab ( $5 \mathrm{mg} / \mathrm{kg}$ BW) $24 \mathrm{~h}$ after the administration of TNBS. On day 4, the BW of all the rats was recorded, and they were anaesthetised intramuscularly with ketamine-xylazine solution (80 and $16 \mathrm{mg} / \mathrm{kg}$ BW, respectively) in normal saline. Subsequently, after incision into the peritoneal cavity, $6 \mathrm{~cm}$ of the colon was removed from immediately above the rectum. Each specimen was separated into three parts and stored for the (1) assessment of histology, (2) evaluation of inflammation and (3) evaluation of oxidative stress. At the completion of the experimental period, the rats were killed by the administration of dolethal directly into the right ventricle of the heart (0.6 mg/kg BW). 


\section{Histological analysis}

Colonic samples $(2-5 \mathrm{~cm})$ were fixed in $10 \%$ buffered formalin. Representative sections were collected every $5 \mathrm{~mm}$, deliberately including areas with any visually apparent damage. The sections were embedded after dehydration into paraffin blocks, and tissue sections ( $4-5 \mu \mathrm{m}$ thick) were stained with haematoxylin and eosin. The samples were examined blindly for histological damage by two pathologists according to a previously described protocol ${ }^{(18)}$, and the following criteria were assessed: (0) architectural damage $0 \cdot 0=$ no abnormality, $0 \cdot 1=$ mild abnormality, $0 \cdot 2=$ moderate diffuse or multifocal abnormalities, and $0.3=$ severe diffuse or multifocal abnormalities; (1) chronic inflammatory infiltrate $-1 \cdot 0=$ no increase, $1 \cdot 1=$ mild but unequivocal increase, $1 \cdot 2=$ moderate increase, and $1 \cdot 3=$ marked increase; $(2)$ eosinophils and neutrophils $-2 \cdot 0=$ no increase, $2 \cdot 1=$ mild but unequivocal increase, $2 \cdot 2=$ moderate increase, and $2 \cdot 3=$ marked increase; (3) neutrophils in epithelium $-3 \cdot 0=$ none, $3 \cdot 1=<5 \%$ crypts involved, $3 \cdot 2=<50 \%$ crypts involved, and $3 \cdot 3=>50 \%$ crypts involved; (4) crypt destruction $4 \cdot 0=$ none, $4 \cdot 1=$ probable local excess of neutrophils in part of the crypt, $4 \cdot 2=$ probable marked attenuation, and $4 \cdot 3=$ unequivocal crypt destruction; (5) erosion or ulceration $-5 \cdot 0=$ none, $5 \cdot 1=$ recovering epithelium + adjacent inflammation; $5 \cdot 2=$ probable erosion focally stripped, $5 \cdot 3=$ unequivocal erosion, and $5 \cdot 4=$ ulcer or granulation tissue.

\section{Colonic tissue homogenisation and total protein quantification}

The tissue samples were weighed and homogenised with PBS ( $1 \mathrm{ml} / 2 \mathrm{~g}$ tissue) and butylated hydroxytoluene $(10 \mu \mathrm{l} / \mathrm{ml}$ homogenised tissue) with an Ultra-Turrax (IKA-Labortechnik) homogeniser. Butylated hydroxytoluene was added to prevent the self-oxidation of samples during homogenisation. Centrifugation was carried out $\left(3000 \mathrm{rpm}\right.$ at $\left.4^{\circ} \mathrm{C}\right)$ for $15 \mathrm{~min}$ to obtain the tissue extract, which was then kept at $-80^{\circ} \mathrm{C}$ until assay. The total protein content was quantified by the Bradford assay $^{(19)}$.

\section{Evaluation of inflammation}

The inflammatory state of the tissue homogenates was assessed through the measurement of ICAM-1, IL-6, IL-8 and IL-10 levels according to the instructions of the ELISA kit manufacturer (R\&D Systems). Their concentrations are expressed as $\mathrm{pg} / \mathrm{mg}$ of total protein.

\section{Assessment of oxidative stress}

Oxidative stress was evaluated by measuring malondialdehyde and GST levels, according to the manufacturer's instructions for their respective assays (OxisResearch and IBL International). Results for malondialdehyde are expressed as $\mathrm{pmol} / \mathrm{mg}$ of total protein, while GST activity is expressed as $\mathrm{nmol} / \mathrm{min}$ per $\mathrm{ml}$.
Table 3. Histological damage scores in healthy and colitic rats $4 \mathrm{~d}$ after the administration of trinitrobenzene sulphonic acid (TNBS)

(Mean values with their standard errors)

\begin{tabular}{llc}
\hline Groups & Colonic damage score & SEM \\
\hline A & 0.088 & 0.048 \\
B & $1.9377^{\star}$ & 0.251 \\
C & 2.375 & 0.132 \\
D & 1.650 & 0.226 \\
E & 1.125 & 0.245 \\
\hline
\end{tabular}

A control, healthy, untreated rats; B, TNBS-induced colitic rats; C, TNBS-induced colitic rats fed Elemental 028 Extra $^{\circledR} ;$ D, TNBS-induced colitic rats fed Emsogen ${ }^{\circledR}$; E, TNBS-induced colitic rats administered infliximab.

* Mean value was significantly different from that of group $\mathrm{A}(P=0.0001)$.

\section{Statistical analysis}

The results are expressed as means with their standard errors. Statistical analysis was carried out by applying the statistical package: SPSS Statistics, version 17.0 (SPSS, Inc.). For comparisons of means, one-way ANOVA with Bonferroni's post hoc analysis was applied. Non-parametric data were analysed with the Kruskal-Wallis test. A $P$ value $<0.05$ was set as the level to determine significance.

\section{Results}

\section{Macroscopic observations and body weight}

Macroscopically, TNBS induced changes in stool consistency, with softer stools seen in group B compared with group A. No significant changes in food intake or in BW were observed between the groups as recorded on days $-12,0$ and 4 .

\section{Histological assessment}

The histological evaluation of the colonic samples revealed a significant difference between the histological damage scores in groups $\mathrm{A}$ and $\mathrm{B}$, confirming the induction of inflammation in the rat model used in the present study $(P=0 \cdot 0001)$. The dietary treatment of rats in group $\mathrm{C}$ elicited a numerical increase in damage scores compared with that of rats in group B, but this was not statistically significant. No significant differences were observed between rats in group $B$ and those in group $\mathrm{D}$ or $\mathrm{E}$, but in these rats, the histological damage scores were numerically lower than those of the TNBS-alone rats in group $\mathrm{B}$ (Table 3 ). The results of the immunohistochemical analysis are shown in Fig. 1.

\section{Inflammatory markers}

As expected, the inflammatory markers in group A were at low background levels. In group B, IL-6 (114 (SEM 11) $v .65$ (sem 6) pg/mg protein), IL-8 (62 (sEM 4) v. 22 (SEM 6) pg/mg protein) and ICAM-1 (10 104 (SEM 412) $v .4483$ (SEM 74) pg/mg protein) levels were elevated, significantly so in the case of ICAM-1 ( $P=0 \cdot 0001)$, compared with those in group A (Fig. 2). 

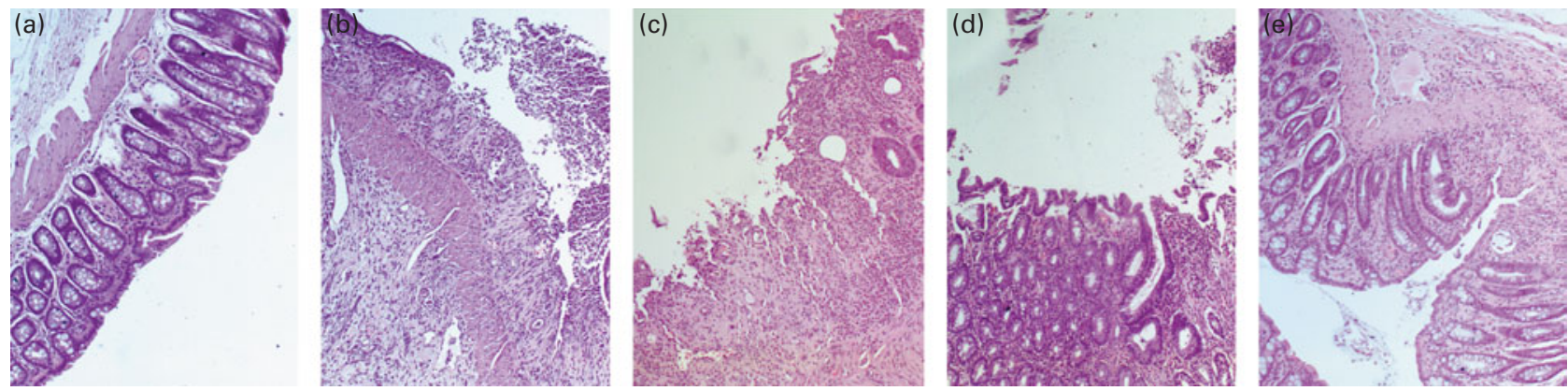

Fig. 1. Results of the immunohistochemical analysis of sections prepared from colonic tissues (125x original magnification). Pictures are representative of microscopic features in individual groups: (a) group A - healthy colonic mucosa; (b) group B - mucosal ulceration, fibrinopurulent exudate, and crypt destruction; (c) group C - chronic and active inflammation in the dermis and epithelium, ulceration, and crypts destruction; (d) group D - ulceration, crypt destruction, and presence of neutrophils and eosinophils and (e) group E - moderate chronic inflammation and presence of neutrophils. (A colour version of this figure can be found online at http://www.journals.cambridge.org/bjn)

The levels of the immunoregulatory cytokine IL-10 were significantly lower in group B than in group A (1.5 (SEM 0.3$) v$. 6 (sEm 0.8) $\mathrm{pg} / \mathrm{mg}$ protein; $P=0 \cdot 001$ ).

In group $\mathrm{C}$, ICAM-1 levels were significantly lower than those in group B (4211 (SEM 167) v. 10104 (SEM 412) pg/mg protein; $P=0.0001)$. In group $\mathrm{D}, \mathrm{IL}-6, \mathrm{IL}-8$ and ICAM-1 (72 (sem 2) v. 114 (SEM 11) $\mathrm{pg} / \mathrm{mg}$ protein, $P=0 \cdot 034 ; 5$ (sem 1) $v$. 62 (SEM 4) $\mathrm{pg} / \mathrm{mg}$ protein, $P=0.0001 ; 3306$ (SEM 170) $v$. 10104 (SEM 412) $\mathrm{pg} / \mathrm{mg}$ protein, $P=0.0001$, respectively) levels were significantly lower than those in group B. Rats in group E had significantly lower IL-8 and ICAM-1 levels

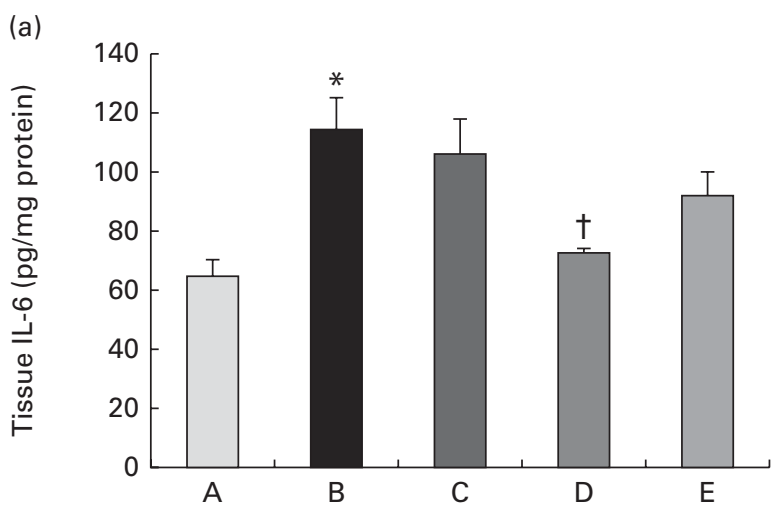

(c)

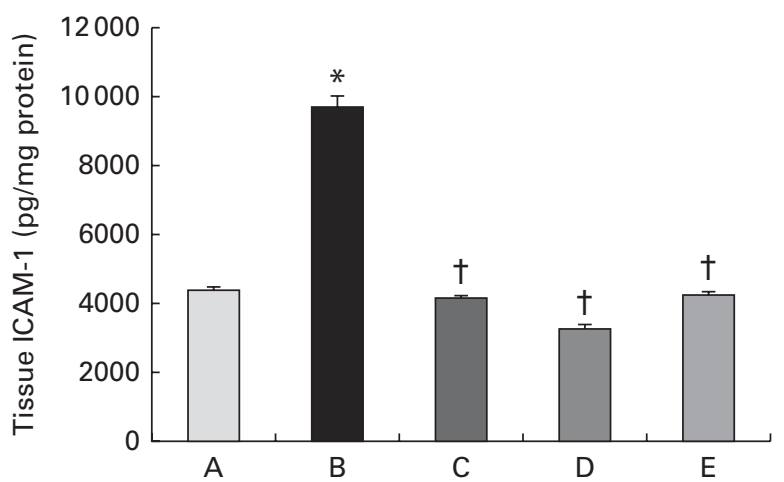

(26 (sem 3) v. 62 (sem 4) pg/mg protein, $P=0 \cdot 032 ; 4356$ (sem 122) v. 10104 (SEM 412) pg/mg protein, $P=0.0001$ ) than those in group $B$ and had significantly higher IL-10 levels (6 (SEM 0.6) v. 1.5 (SEM 0.3) pg/mg protein; $P=0.0001)$.

\section{Markers of oxidative stress}

Malondialdehyde levels in groups C, D and E were numerically lower than those in group B, but no significant differences were observed (Fig. 3). Rats in group B rats exhibited much higher GST activity than those in group A (74 (SEM 1.7) $v .1 \cdot 7$

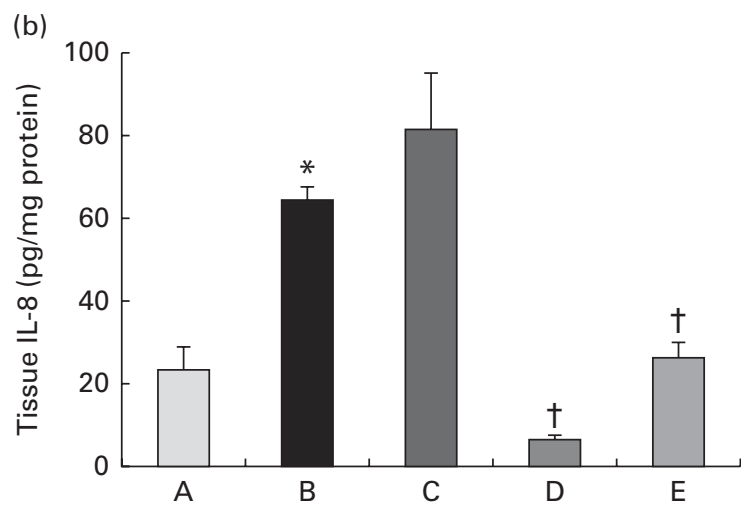

(d)

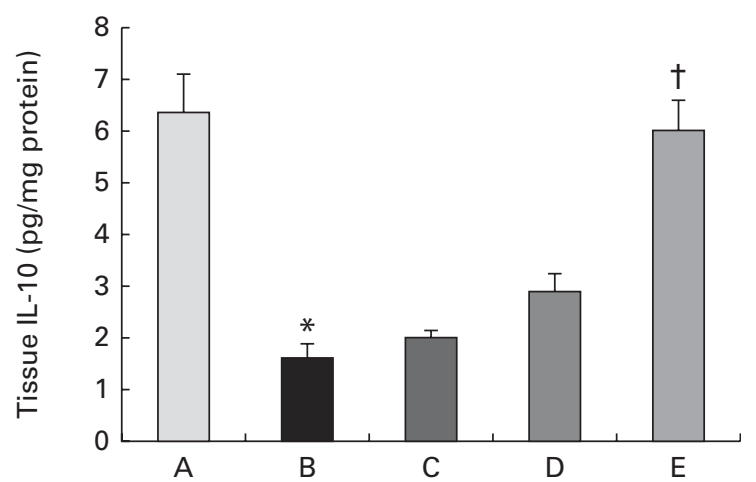

Fig. 2. Inflammatory markers in colonic tissue homogenates. (a) Levels of IL-6, (b) levels of IL-8, (c) levels of intercellular adhesion molecule-1 (ICAM-1) and (d) levels of IL-10. Values are means, with their standard errors represented by vertical bars. * Mean values were significantly different from those of group A $(P<0.05)$. † Mean values were significantly different from those of group B $(P<0.05)$. A, control, healthy, untreated rats; B, TNBS-induced colitic rats; C, TNBS-

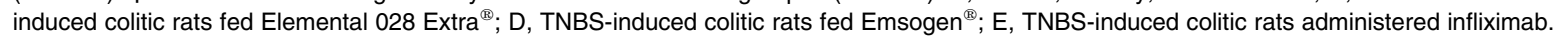



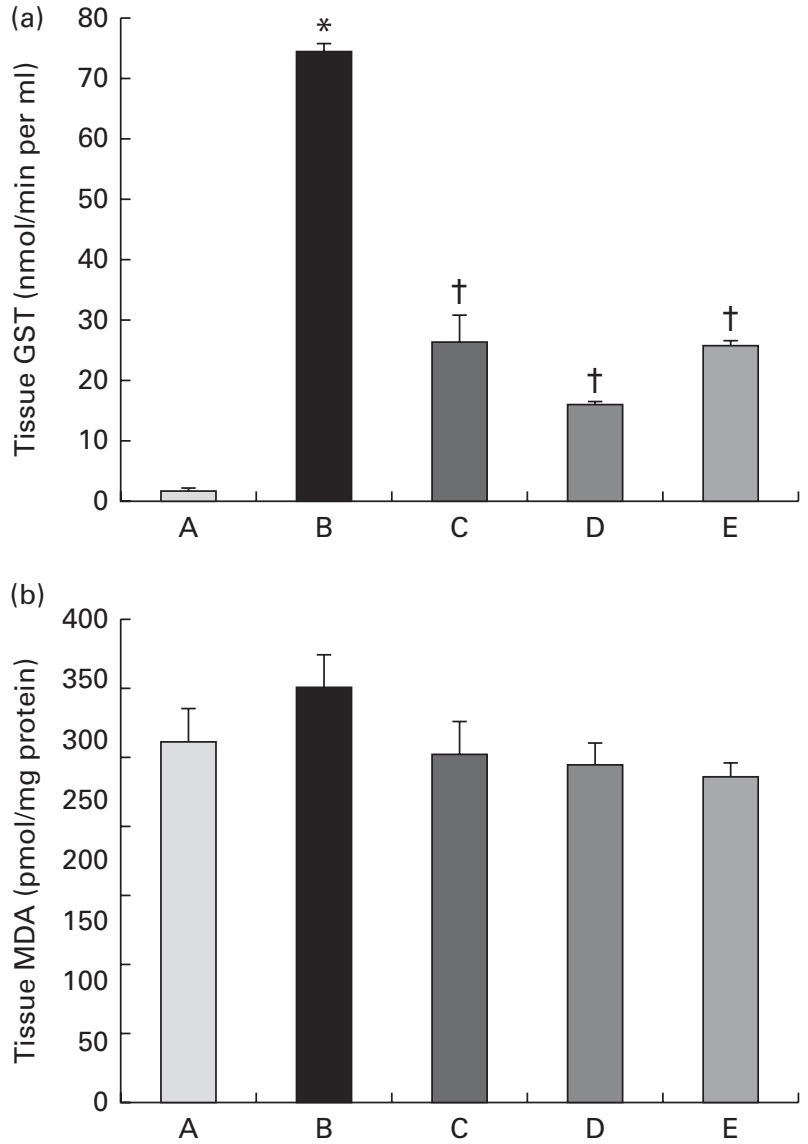

Fig. 3. Oxidative stress markers in colonic tissue homogenates. (a) Levels of glutathione $S$-transferase (GST) and (b) levels of malondialdehyde (MDA). Values are means, with their standard errors represented by vertical bars. ${ }^{*}$ Mean values were significantly different from those of group A $(P<0.05)$. † Mean values were significantly different from those of group $\mathrm{B}$ $(P<0.05)$. A, control, healthy, untreated rats; $\mathrm{B}$, TNBS-induced colitic rats; $\mathrm{C}$, TNBS-induced colitic rats fed Elemental 028 Extra ${ }^{\circledR} ;$ D, TNBS-induced colitic rats fed Emsogen ${ }^{\circledR}$; E, TNBS-induced colitic rats administered infliximab.

$(\operatorname{SEM} 0 \cdot 2) \mathrm{nmol} / \mathrm{min}$ per $\mathrm{ml} ; P=0 \cdot 0001)$. GST activity was significantly less in groups $\mathrm{C}, \mathrm{D}$ and $\mathrm{E}$ than in group $\mathrm{B}(26 \cdot 2$ (SEM 4.5) v. $74(\operatorname{sem~} 1.7) \mathrm{nmol} / \mathrm{min}$ per $\mathrm{ml}, P=0.0001 ; 15.8$ (SEM 0.6) $v .74(\operatorname{sem~} 1.7) \mathrm{nmol} / \mathrm{min}$ per $\mathrm{ml}, P=0 \cdot 0001 ; 19 \cdot 2($ SEM $0 \cdot 6) v .74$ $($ SEM 1.7$) \mathrm{nmol} / \mathrm{min}$ per $\mathrm{ml}, P=0.0001 ; 25.6$ (SEM 1) $v .74$ (SEM $1.7) \mathrm{nmol} / \mathrm{min}$ per $\mathrm{ml}, P=0.0001$, respectively).

\section{Discussion}

As the standard medical management of IBD includes numerous side effects and is not always effective, there is an increasing interest in the possible anti-inflammatory effects of natural products such as dietary constituents ${ }^{(20,21)}$ and EN. The optimal fat content of EN formulae remains a controversial issue, not least since the type of fat may interact differentially when there are polymorphisms in the cytokines regulating disease activity ${ }^{(22)}$. Thus, the objective of the present study was to evaluate the efficacy of two EN formulae with differing fat content in a rat model of colitis.

The results show that a formula rich in MCT ameliorates TNBS-induced colitis in rats. Treatment with the MCT-rich diet yielded significantly less increase in IL-6, IL-8 and ICAM-1 levels and GST activity, an effect comparable to that of infliximab. The LCT-rich diet did not show such an activity and suppressed only ICAM-1 levels and GST activity. Although the histological data were not so compelling, the present results indicate that an EN formula rich in MCT may be more beneficial than other formulae in IBD.

The TNBS-colitis model ${ }^{(23)}$ is self-evidently not identical to human IBD, but its similarity and robustness have led to its widespread use in the investigation of IBD pathogenesis and in the assessment of new potential therapeutic agents. In this model, ethanol increases intestinal permeability so that TNBS reaches the subepithelial space. The resulting severe inflammatory response disrupts $\mathrm{T}$-cell tolerance to mucosal antigens and alters T-cell localisation in the lamina propria, intraepithelial space and lymphoreticular tissues. TNBS colitis is characterised by macrophage, polymorphonuclear leucocyte and lymphocyte infiltration and ulceration ${ }^{(23)}$ There is CD-like intestinal inflammation with the secretion of IL-1, IL-6, IL-12, IL-18, TNF- $\alpha$ and interferon- $\gamma$ by activated $\mathrm{T}$ helper 1 cells and of IL-4, IL- 5 and IL- 13 by T helper 2 cells ${ }^{(24)}$. The significant differences in inflammatory markers and the histological damage scores in the TNBS-induced colitis group compared with those in the control group confirm the effective induction of TNBS colitis in the present study.

The levels of IL-6, which promotes apoptosis resistance and $\mathrm{T}$-cell proliferation in the lamina propria, are significantly increased in the serum of CD patients ${ }^{(25)}$. A pilot trial of a humanised monoclonal antibody to the IL-6 receptor in active $C D$ patients has demonstrated an increase in the response rate in the treated group compared with that in the placebo group ${ }^{(26)}$. In the present study, the MCT-rich diet, but not the LCT-rich diet (which has much less MCT), induced a significant relative decrease in IL-6 levels, indicating that the MCT content may be the key factor. Notably, this observation exceeds the effect observed with infliximab. The levels of IL-8, a chemokine responsible for the accumulation and stimulation of polymorphonuclear neutrophils at sites of inflammation ${ }^{(27)}$ and which is overexpressed in patients with active $\mathrm{CD}^{(28)}$, were also suppressed by the MCT-rich diet.

The levels of ICAM-1, a molecule promoting the adhesion between leucocytes and endothelial cells and present in higher concentrations in $\operatorname{IBD}^{(29,30)}$, were suppressed by all the anti-inflammatory strategies used in the present study, but dietary therapy seemed less able to yield an IL-10 immunoregulatory response.

Oxidative stress has been suggested as a possible aetiological factor in IBD pathogenesis ${ }^{(31-35)}$. We found no direct evidence to support this from our model. However, induction of GST activity was observed and its suppression by each of the anti-inflammatory strategies was demonstrated.

Although the mechanisms underlying the efficacy of elemental formulae include the exclusion of food antigens and change of the colonic bacterial environment ${ }^{(36)}$, the contrasting effects of two diets differing mainly in their lipid composition suggest that the nature of the dietary fat is also important, first proposed by Fernández-Bañares et al. ${ }^{(37)}$. The MCT-rich diet exerted stronger anti-inflammatory effects 
than the LCT-rich diet, which may be explained by the leucocyte immunomodulatory properties of $\operatorname{MCT}^{(38,39)}$. MCT is known to regulate mucosal damage and inflammation ${ }^{(14,15,17,40)}$. Two independent surveys have shown a remarkable inverse correlation between remission rates in active $\mathrm{CD}$ and the LCT content of elemental feeds ${ }^{(11,41)}$. Animal studies have shown that LCT absorption is associated with enhanced migration of lymphocytes, up-regulation of adhesion molecule levels and stimulation of lymphocyte function ${ }^{(42)}$. A meta-analysis supports the absence of a difference in inflammatory response between groups with low and high LCT content ${ }^{(12)}$. The trial carried out by Leiper et al. ${ }^{(43)}$ showed no difference in the effect of low-LCT and high-LCT whole-protein feeds in active $\mathrm{CD}$, leading to the hypothesis that all previously reported correlations between the LCT content of the diet and response rates in active CD are unlikely to be due to LCT itself and may be due to some other component of high-LCT feeds. It is highly probable that the effects reported herein are a consequence of the MCT content of the diet.

It is acknowledged that our model differs from the clinical context in that the experimental diets were administered before, as well as after, the induction of IBD. However, this is an established methodology when aiming to evaluate the effectiveness of diet in the TNBS-colitis model, also given that the effect of TNBS diminishes rapidly within $4-5 \mathrm{~d}$ of administration. An interpretation that MCT is acting primarily in a prophylactic capacity receives support from another rat study (of MCT and $n-3$ PUFA) ${ }^{(17)}$, but unguarded extrapolation should obviously be avoided.

The MCT-rich diet also has a higher $n-6: n-3$ PUFA ratio. Excess $n-6$ lipids have traditionally been considered to exacerbate inflammation, and high levels have been reported to be positively correlated with the CD activity index ${ }^{(44)}$. Nevertheless, there are several studies in rat models of colitis that fail to demonstrate significant negative effects of a high $n-6: n-3$ PUFA ratio ${ }^{(45-47)}$. The content of EPA and DHA is also important when considering the effectiveness of PUFA-rich diets in the inflammatory response. However, so far, the majority of studies investigating the anti-inflammatory effects of $n-3$ PUFA have utilised fish oils, with a heterogeneous mixture of EPA and DHA, and only a small number of studies have shown that pure DHA and/or EPA can reduce inflammation in vitro ${ }^{(48)}$ or in experimental animals ${ }^{(49)}$. The precise content of DHA and EPA in the feeds that we used has not been established with confidence (it is not present in the product labelling), and any hypothesis on their effects would be speculative.

However, it would be oversimplistic to attribute benefits only to MCT, as alteration of the SCFA and MUFA may also be important ${ }^{(50,51)}$. Indeed, our own, recent animal data ${ }^{(47)}$ have shown that intravenous infusions of a MUFA-rich lipid emulsion increase IL-6 levels and induce more histological damage than MCT- and n-6 PUFA-rich emulsions. The MCT emulsion had the most desirable effects. Additionally, a major clinical trial $^{(52)}$ in patients with active $\mathrm{CD}$ has demonstrated significantly lower remission rates after the consumption of an enteral formula rich in MUFA than after that of a formula rich in linoleic acid (15\% of total energy in the LCT-rich diet compared with $1 \%$ in the MCT-rich diet).

The apparent beneficial impact of a diet rich in MCT, low in MUFA and relatively low in PUFA (either $n-3$ or $n$-6) in TNBS colitis supports the premise that modified fat EN may be a more effective choice for IBD patients than a standard feed. However, current guidance on therapeutic nutrition considers only Crohn's disease and, even there, places almost all of the emphasis on the treatment of small bowel disease. The intrinsic properties of the TNBS model suggest that dietary evaluation in Crohn's colitis and indeed in ulcerative colitis should also be revisited.

\section{Acknowledgements}

The authors thank the Experimental Research Unit of ELPENPharmaceuticals for animal breeding and husbandry.

A. F. acknowledges support from the Biomedical Research Centre funding of UCL and its partner hospitals from the UK National Institute for Health Research. Biomedical Research Centre funding of UCL and partner hospitals from the UK National Institute for Health Research had no role in the design, analysis or writing of this article.

The authors' contributions are as follows: E. P. conducted the experimental and statistical analyses and wrote the first draft of the manuscript; A. C. K. and A. F. designed the protocol and edited the manuscript; A. G. and A. P. conducted the experimental analysis and contributed to the interpretation of the results. All the authors contributed substantially to the present study.

None of the authors has any conflicts of interest to declare.

\section{References}

1. Lakatos PL (2006) Recent trends in the epidemiology of inflammatory bowel diseases: up or down? World J Gastroenterol 12, 6102-6108.

2. Loftus EV (2004) Clinical epidemiology of inflammatory bowel disease: incidence, prevalence, and environmental influences. Gastroenterology 126, 1504-1517.

3. Viscido A, Aratari A, Maccioni F, et al. (2005) Inflammatory bowel diseases: clinical update of practical guidelines. $\mathrm{Nucl}$ Med Commun 26, 649-655.

4. Bouma G \& Strober W (2003) The immunological and genetic basis of inflammatory bowel disease. Nat Rev Immunol 3, 521-533.

5. D'Odorico A, Bortolan S, Cardin R, et al. (2001) Reduced plasma antioxidant concentrations and increased oxidative DNA damage in inflammatory bowel disease. Scand $J$ Gastroenterol 36, 1289-1294.

6. Beltrán B, Nos P, Dasí F, et al. (2010) Mitochondrial dysfunction, persistent oxidative damage and catalase inhibition in naive and treated Crohn's disease. Inflamm Bowel Dis 16, $76-86$.

7. Pithadia $\mathrm{AB} \&$ Jain S (2011) Treatment of inflammatory bowel disease (IBD). Pharmacol Rep 63, 629-642.

8. Feagan BG, Lémann M, Befrits R, et al. (2012) Recommendations for the treatment of Crohn's disease with tumor necrosis factor antagonists: an expert consensus report. Inflamm Bowel Dis 18, 152-160. 
9. Forbes A, Goldesgeyme E \& Paulon E (2011) Nutrition in inflammatory bowel disease. J Parenter Enteral Nutr 35 , 571-580.

10. Bamba T, Shimoyama T, Sasaki M, et al. (2003) Dietary fat attenuates the benefits of an elemental diet in active Crohn's disease: a randomized, controlled trial. Eur J Gastroenterol Hepatol 15, 151-157.

11. Middleton SJ, Rucker JT, Kirby GA, et al. (1995) Long-chain triglycerides reduce the efficacy of enteral feeds in patients with active Crohn's disease. Clin Nutr 14, 229-236.

12. Zachos M, Tondeur M \& Griffiths AM (2007) Enteral nutritional therapy for induction of remission in Crohn's disease. Cochrane Database Systematic Review CD000542.

13. Andoh A, Takaya H, Araki Y, et al. (2000) Medium- and long-chain fatty acids differentially modulate interleukin-8 secretion in human fetal intestinal epithelial cells. $J$ Nutr 130, 2636-2640.

14. Tsujikawa T, Ohta N, Nakamura T, et al. (1999) Mediumchain triglycerides modulate ileitis induced by trinitrobenzene sulfonic acid. J Gastroenterol Hepatol 14, 1166-1172.

15. Tsujikawa T, Ohta N, Nakamura T, et al. (2001) Mediumchain triglyceride-rich enteral nutrition is more effective than low-fat enteral nutrition in rat colitis, but is equal in enteritis. J Gastroenterol 36, 673-680.

16. Hassan A, Ibrahim A, Mbodji K, et al. (2010) An $\alpha$-linolenic acid-rich formula reduces oxidative stress and inflammation by regulating NF- $\mathrm{\kappa B}$ in rats with TNBS-induced colitis. J Nutr 140, 1714-1721

17. Kono H, Fujii H, Ogiku M, et al. (2010) Enteral diets enriched with medium-chain triglycerides and $n-3$ fatty acids prevent chemically induced experimental colitis in rats. Transl Res 156, 282-291.

18. Geboes K, Riddell R, Ost A, et al. (2000) A reproducible grading scale for histological assessment of inflammation in ulcerative colitis. Gut 47, 404-409.

19. Bradford MM (1976) A rapid and sensitive method for the quantitation of microgram quantities of protein utilizing the principle of protein-dye binding. Ann Biochem 72, 248-254.

20. Kaliora AC, Stathopoulou MG, Triantafillidis JK, et al. (2007) Chios mastic treatment of patients with active Crohn's disease. World J Gastroenterol 13, 748-753.

21. Kwon HK, Hwang JS, Lee CG, et al. (2011) Cinnamon extract suppresses experimental colitis through modulation of antigen-presenting cells. World J Gastroenterol 17, 976-986.

22. Guerreiro CS, Ferreira P, Tavares L, et al. (2009) Fatty acids, IL-6, and TNF-alpha polymorphisms: an example of nutrigenetics in Crohn's disease. Am $J$ Gastroenterol 104, 2241-2249.

23. Morris GP, Beck PL, Herridge MS, et al. (1989) Hapteninduced model of chronic inflammation and ulceration in the rat colon. Gastroenterology 96, 795-803.

24. Velde A, Verstege M \& Hommes D (2006) Critical appraisal of the current practice in murine TNBS-induced colitis. Inflamm Bowel Dis 12, 995-999.

25. Mudter J \& Neurath FM (2007) IL-6 signaling in inflammatory bowel disease: pathophysiological role and clinical relevance. Inflamm Bowel Dis 13, 1016-1023.

26. Ito H, Takazoe M, Fukuda Y, et al. (2004) A pilot randomized trial of a human anti-interleukin-6 receptor monoclonal antibody in active Crohn's disease. Gastroenterology 126, 989-996.

27. Ina K, Kusugami K, Yamaguchi T, et al. (1997) Mucosal interleukin-8 is involved in neutrophil migration and binding to extracellular matrix in inflammatory bowel disease. $\mathrm{Am} \mathrm{J}$ Gastroenterol 92, 1342-1346.
28. MacDermott RP (1999) Chemokines in the inflammatory bowel diseases. J Clin Immunol 19, 266-272.

29. Danese S, de la Motte C, Sturm A, et al. (2003) Platelets trigger a CD40-dependent inflammatory response in the microvasculature of inflammatory bowel disease patients Gastroenterology 124, 1249-1264.

30. Nielsen $\mathrm{OH}$, Langholz E, Hendel J, et al. (1994) Circulating soluble intercellular adhesion molecule-1 (sICAM-1) in active inflammatory bowel disease. Dig Dis Sci 39 1918-1923.

31. Kruidenier L \& Verspaget HW (2002) Oxidative stress as a pathogenic factor in inflammatory bowel disease: radicals or ridiculous? Aliment Pharmacol Ther 16, 1997-2015.

32. Kruidenier L, Kuiper I, Van Duijn W, et al. (2003) Imbalanced secondary mucosal antioxidant response in inflammatory bowel disease. $J$ Pathol 201, 17-27.

33. Tüzün A, Erdil A, Inal V, et al. (2002) Oxidative stress and antioxidant capacity in patients with inflammatory bowel disease. Clin Biochem 35, 569-572.

34. Ademoglu E, Erbil Y, Tam B, et al. (2004) Do vitamin E and selenium have beneficial effects on trinitrobenzenesulfonic acid-induced experimental colitis. Dig Dis Sci 49, 102-108.

35. Girgin F, Karaoglu O, Erkuş M, et al. (2000) Effects of trimetazidine on oxidant/antioxidant status in trinitrobenzenesulfonic acid-induced chronic colitis. I Toxicol Environ Health A 59, 641-662.

36. Shah S (2007) Dietary factors in the modulation of inflammatory bowel disease activity. MedGenMed 9,60.

37. Fernández-Bañares F, Cabré E, González-Huix F, et al. (1994) Enteral nutrition as primary therapy in Crohn's disease. Gut 34, Suppl. 1, S55-S59.

38. Waitzberg DL, Bellinati-Pires R, Yamaguchi N, et al. (1996) Influence of medium-chain triglyceride-based lipid emulsion on rat polymorphonuclear cell functions. Nutrition $\mathbf{1 2}$ 93-99.

39. Wanten GJ, Geijtenbeek TB, Raymakers RA, et al. (2000) Medium-chain triglyceride-containing lipid emulsions increase human neutrophil b2 integrin expression, adhesion, and degranulation. J Parenter Enteral Nutr 24, 228-233.

40. Ohta N, Tsujikawa T, Nakamura T, et al. (2003) A comparison of the effects of medium- and long-chain triglycerides on neutrophil stimulation in experimental ileitis. J Gastroenterol 38, 127-133.

41. Evans RC \& Rhodes JM (1996) Understanding the mechanism of dietary therapy in Crohn's disease - meta-analysis of polymeric enteral feeding studies and pilot study of maintenance with low fibre/low fat diet. Gut 38, A637.

42. Miura S, Tsuzuki Y, Hokari R, et al. (1998) Modulation of intestinal immune system by dietary fat intake: relevance to Crohn's disease. J Gastroenterol Hepatol 13, 1183-1190.

43. Leiper K, Woolner J, Mullan MM, et al. (2001) A randomised controlled trial of high versus low long chain triglyceride whole protein feed in active Crohn's disease. Gut 49, 790-794.

44. Kuroki F, Iida M, Matsumoto T, et al. (1997) Serum n3 polyunsaturated fatty acids are depleted in Crohn's disease. Dig Dis Sci 42, 1137-1141.

45. Ramakers JD, Mensink RP, Verstege MI, et al. (2008) An arachidonic acid-enriched diet does not result in more colonic inflammation as compared with fish oil- or oleic acid-enriched diets in mice with experimental colitis Br J Nutr 100, 347-354

46. Vieira de Barros K, Gomes de Abreu G, Xavier RA, et al (2011) Effects of a high fat or a balanced omega 3/omega 6 diet on cytokines levels and DNA damage in experimental colitis. Nutrition 27, 221-226. 
47. Bertevello PL, De Nardi L, Torrinhas RS, et al. (2012) Partial replacement of $\omega-6$ fatty acids with medium-chain triglycerides, but not olive oil, improves colon cytokine response and damage in experimental colitis. I Parenter Enteral Nutr 36, 442-448.

48. Ibrahim A, Mbodji K, Hassan A, et al. (2011) Antiinflammatory and anti-angiogenic effect of long chain $n$-3 polyunsaturated fatty acids in intestinal microvascular endothelium. Clin Nutr 30, 678-687.

49. Cho JY, Chi S-G \& Chun HS (2011) Oral administration of docosahexaenoic acid attenuates colitis induced by dextran sulfate sodium in mice. Mol Nutr Food Res 55, 239-246.
50. Hou JK, Abraham B \& El-Serag H (2011) Dietary intake and risk of developing inflammatory bowel disease: a systematic review of the literature. Am J Gastroenterol 106, 563-573.

51. Sakamoto N, Kono S, Wakai K, et al. (2004) Dietary risk factors for inflammatory bowel disease: a multicenter casecontrol study in Japan. Inflamm Bowel Dis 11, 154-163.

52. Gassull MA, Fernandez-Banares F, Cabre E, et al. (2002) Fat composition may be a clue to explain the therapeutic effect of enteral nutrition in Crohn's disease: results of a double blind randomised multicentre European trial. Gut 51, 164-168. 